\title{
Hydrogen and helium emission of the symbiotic binary AG Draconis during an active phase $(1996-1997)^{\star}$
}

\author{
N. Tomov and M. Tomova
}

\begin{abstract}
Institute of Astronomy and Isaac Newton Institute of Chile Bulgarian Branch, Bulgarian National Astronomical Observatory, PO Box 136, 4700 Smolyan, Bulgaria
\end{abstract}

Received 7 November 2001 / Accepted 13 March 2002

\begin{abstract}
High resolutions observations of the regions of the $\mathrm{H} \alpha$ and He II 4868 lines of the symbiotic star AG Dra were performed during its time of activity in 1996-1997. Profiles, fluxes and radial velocity data of these lines as well as the line HeI 4713 were obtained. The $\mathrm{H} \alpha$ fluxes changed in correlation with the optical light. The emission measure of the circumbinary nebula of the system at several epochs during its 1994-1998 active phase was also calculated using Balmer continuum emission on the basis of $U$ photometric data from the literature. It was increased by factors up to 36 above its quiescent maximal value. After the 1996 and 1997 light maxima the profile of the HeII 4868 line contained a broad emission component indicating mainly a stellar wind from the hot secondary with a velocity of $1100 \div 1300 \mathrm{~km} \mathrm{~s}^{-1}$. The mass-loss rate of this star was estimated to be about $1 \div 210^{-7}(\mathrm{~d} / 1.7 \mathrm{kpc})^{3 / 2} M_{\odot} \mathrm{yr}^{-1}$. The growth of its Lyman luminosity however is not sufficient to explain the increased nebular emission during the active phase. A view that this emission could be the result of both a rise of the mass-loss rate of the cool giant of the system and a rise of the Lyman flux of the hot luminous secondary, being in a steady-state burning regime during the quiescence, is discussed.
\end{abstract}

Key words. stars: binaries: symbiotic - stars: activity - stars: individual: AG Dra - stars: mass-loss - line: profiles

\section{Introduction}

Symbiotic stars are interpreted as interacting binary systems consisting of a cool luminous visual primary and a secondary component which is a hot compact object or a main sequence star. The components' interaction is realized via transfer of mass from the atmosphere of the giant star and its accretion onto the secondary component. Accretion is the mechanism that gives rise to the secondary's physical activity. In a number of cases this activity leads to an unexpected rapid increase in the visual light of 3-4 mag followed by slow decrease to its previous value for months or years. The symbiotic system AG Dra consists of a high luminosity K giant (Mikolajewska et al. 1995; Smith et al. 1996; Friedjung et al. 1998) and a hot compact object whose temperature is probably more than $10^{5} \mathrm{~K}$ (Greiner et al. 1997). In 1994 it entered in a new

Send offprint requests to: N. Tomov,

e-mail: rozhen@mbox.digsys.bg

* Based on observations collected at the National Astronomical Observatory Rozhen, Bulgaria. active phase characterized by some consecutive outbursts having intervals of about one year between the times of their light maxima and $U$ amplitudes up to more than $3 \mathrm{mag}$. In this way AG Dra underwent five optical brightenings, the last of them being in 1998. Analysis of the photometric data (Montagni et al. 1996; Greiner et al. 1997; Skopal 1998; Petrik et al. 1998; Tomova \& Tomov 1998; Tomov \& Tomova 2000) showed that the growth of the visual light is due to the increased radiation of the circumbinary nebula. Different models about the outburst activity of AG Dra have been proposed.

Leibowitz \& Formiggini (1992) supposed the 1980-1983 and 1985-1986 optical outbursts of AG Dra to be associated with the liberation of mechanical energy in the atmosphere of the giant. Mikolajewska et al. (1995) came to the conclusion that these outbursts are most probably due to thermonuclear events on the surface of a white dwarf. Greiner et al. (1997) assumed the 1994 and 1995 outbursts to be determined by an expansion and cooling of the compact secondary, since their ROSAT data anticorelate with the optical photometry. 
They proposed this star to be in steady-state burning of hydrogen at its surface, which provides its high X-ray luminosity during the quiescent state of the system. The accretion rate increases during the outburst and causes accumulation of matter, which is not included in the burning process. As a result of the expanding and cooling, the star decreases its X-ray emission. One possibility for the expansion to be restricted, according to the authors, is the appearance of a high velocity wind from the hot photosphere. The cooling of the star in principle provides a possibility for growth of its optical continuum but the visual brightenings of the system are caused by variation of its nebular spectrum. That is why the authors are forced to search for an additional emission mechanism in the surrounding nebula.

Gonzalez-Riestra et al. (1999) identified cool and hot outbursts of the AG Dra system determining the Zanstra temperature of its hot component. During the cool 1980-1983 and 1994-1995 outbursts the temperature of this component decreased compared to its quiescent value and during the hot 1985-1986 outbursts it increased. This means that the mechanisms driving the activity of this symbiotic binary are probably more than one.

Galis et al. (1999) proposed a mechanism for increase of the accretion rate related to resonance of the pulsations of the cool giant and its orientation to the companion. They supposed that the pulsations are probably nonradial, leading to an appearance of an asymmetric stellar wind. The ratio of the orbital to the pulsation period was found to be very close to $14 / 9$. It is possible for the massloss of the giant to reach its maximal value when the most dense part of the wind is orientated towards the companion. This would provide a great increase of the accretion rate causing the observed outbursts.

One approach to test any single outburst model consists of investigation of the possibility of the observed changes of the emission of the circumbinary nebula being explained in the framework of that model. In this paper we analyse high resolution data in the regions of the $\mathrm{H} \alpha$ and He II 4686 lines of the spectrum of AG Dra acquired during its 1996 and 1997 visual brightenings. In addition we calculate the emission measure of its circumbinary nebula using $U$ photometric data from the literature. We continue our search, initiated in a previous work (Tomova \& Tomov 1999), for observational evidence to support any single outburst model. On one hand the frequency of appearance of a stellar wind from the hot component of the system and its mass-loss rate obtained by us cannot be explained in the framework of the thermonuclear model. On the other hand the mechanism proposed for the interpretation of the X-ray data meets difficulties with the increase of the nebular emission. One of the conclusions of our analysis of the quiescent $U$ photometric data of this system (Tomov et al. 2000) was that its hot component probably has an excess of luminosity, making it able to ionize the greatest part of the nebula with the exception only of the region behind the cool giant. That is why it may be able to ionize additional amount of gas in the wind
Table 1. List of the observations.

\begin{tabular}{llll}
\hline \hline Date & $\begin{array}{l}\text { JD } \\
2450000+\end{array}$ & Phase & $\begin{array}{l}\text { Spectral } \\
\text { region }\end{array}$ \\
\hline 1996 Jul. 28 & 293.321 & 0.082 & $\mathrm{H} \alpha$ \\
1996 Jul. 28 & 293.394 & 0.082 & $\mathrm{He} \mathrm{II} 4686$ \\
1996 Jul. 30 & 295.489 & 0.086 & $\mathrm{H} \alpha$ \\
1996 Aug. 2 & 298.396 & 0.091 & $\mathrm{H} \alpha$ \\
1996 Aug. 25 & 321.383 & 0.133 & $\mathrm{H} \alpha$ \\
1996 Aug. 26 & 322.282 & 0.134 & $\mathrm{He}$ II 4686 \\
1997 Jan. 27 & 475.523 & 0.412 & $\mathrm{H} \alpha$ \\
1997 Mar. 28 & 536.333 & 0.522 & $\mathrm{H} \alpha$ \\
1997 Apr. 26 & 565.383 & 0.574 & $\mathrm{H} \alpha$ \\
1997 Jun. 18 & 618.419 & 0.670 & $\mathrm{H} \alpha$ \\
1997 Jun. 19 & 619.369 & 0.672 & $\mathrm{He} \mathrm{II} 4686$ \\
1997 Jul. 12 & 641.515 & 0.712 & $\mathrm{H} \alpha$ \\
1997 Aug. 13 & 674.340 & 0.772 & $\mathrm{H} \alpha$ \\
1997 Aug. 16 & 677.283 & 0.777 & $\mathrm{He} \mathrm{II} 4686$ \\
1997 Sep. 11 & 703.243 & 0.824 & $\mathrm{H} \alpha$ \\
1997 Sep. 11 & 703.307 & 0.824 & $\mathrm{He}$ II 4686 \\
1997 Sep. 13 & 705.332 & 0.828 & $\mathrm{H} \alpha$ \\
1997 Oct. 10 & 732.274 & 0.877 & $\mathrm{He} \mathrm{II} 4686$ \\
1997 Oct. 10 & 732.356 & 0.877 & $\mathrm{H} \alpha$ \\
\hline
\end{tabular}

of the giant when its mass-loss rate increases during outburst phase. This would be a natural explanation of the growth of the nebular emission. The consideration of this idea forms part of our work.

\section{Observation and reduction}

Nineteen CCD frames were acquired on sixteen nights during 1996 and 1997 with the Coudé spectrograph of the $2 \mathrm{~m}$ RCC telescope of the National Astronomical Observatory Rozhen. Two spectral regions were detected: one near $\mathrm{H} \alpha$ and the other near the He II 4868 line (Table 1). The spectral window of $110 \AA$ gave us the possibility of observing the He I 4713 line together with He II 4868. The resolving power was 15000 . The IRAF package was used for data reduction as well as for obtaining the dispersion curve and calculating the radial velocities and the equivalent widths. The fluxes of the He I 4713 and He II 4868 lines were obtained by means of the equivalent widths and the monochromatic continuum fluxes at their positions. The continuum fluxes were calculated via linear interpolation of the fluxes at the positions of the sensitivity maxima of the $B$ and $V$ photometric systems. The flux of the line $\mathrm{H} \alpha$ can be obtained in the same way using $V$ and $R$ photometric data. However we did not have $R$ photometric data and determined the monochromatic continuum flux at its position as follows.

Two components of the AG Dra system have contributions to its continuum at the position of $\mathrm{H} \alpha$ - the cool giant and the circumbinary nebula. We obtained the flux of the cool giant supposing that it has the same 
Table 2. The $U$ flux and the emission measure of the circumbinary nebula.

\begin{tabular}{|c|c|c|c|c|c|c|c|}
\hline No & Date & $\begin{array}{l}\text { JD } \\
2449000+\end{array}$ & Phase & $\begin{array}{l}U \\
\text { mag }\end{array}$ & $F^{\text {neba }}$ & $E M^{\mathrm{b}}$ & $m$ \\
\hline 1 & 1994 Jul. 16 & 550 & 0.736 & 7.60 & 6.319 & 57.56 & 36.2 \\
\hline 2 & 1995 Mar. 23 & 800 & 0.189 & 9.90 & 0.687 & 6.26 & 3.9 \\
\hline 3 & 1995 Jul. 22 & 921 & 0.408 & 8.70 & 2.249 & 20.49 & 12.9 \\
\hline 4 & 1996 Feb. 6 & 1120 & 0.768 & 10.40 & 0.406 & 3.70 & 2.3 \\
\hline 5 & 1996 May 22 & 1226 & 0.960 & 11.26 & 0.139 & 1.27 & 0.8 \\
\hline 6 & 1996 Jul. 2 & 1267 & 0.034 & 9.15 & 1.455 & 13.26 & 8.3 \\
\hline 7 & 1996 Nov. 12 & 1400 & 0.275 & 11.10 & 0.175 & 1.59 & 1.0 \\
\hline 8 & 1997 Aug. 19 & 1680 & 0.782 & 8.55 & 2.595 & 23.64 & 14.9 \\
\hline 9 & 1998 Jan. 16 & 1830 & 0.054 & 11.56 & 0.086 & 0.78 & 0.5 \\
\hline 10 & 1998 Aug. 1 & 2027 & 0.410 & 9.39 & 1.148 & 10.45 & 6.6 \\
\hline
\end{tabular}

a Continuum fluxes in units $10^{-12} \mathrm{erg} \mathrm{cm}^{-2} \mathrm{~s}^{-1} \AA^{-1}$.

b $E M=n_{\mathrm{e}}^{2} V \times 10^{59}(\mathrm{~d} / 1.7 \mathrm{kpc})^{2} \mathrm{~cm}^{-3}$.

continuum energy distribution as $\alpha$ Boo, since the temperatures of these stars are equal, amounting to $4300 \mathrm{~K}$ (Smith et al. 1996; Griffin \& Lynas-Gray 1999). We calculated the $6563 \AA / 5500 \AA$ flux ratio of $\alpha$ Boo and scaled it to the dereddened visual flux of AG Dra taken in its quiescent orbital minimum, when the nebular emission can be supposed to be negligible.

Both groups of variations of the optical continuum of AG Dra, the quiescent and the active ones, are caused by the nebular emission (Montagni et al. 1996; Greiner et al. 1997; Tomova \& Tomov 1998; Tomov et al. 2000). That is why the contribution of the circumbinary nebula at the wavelength position $\lambda 6563 \AA$ must be found at each epoch of $\mathrm{H} \alpha$ observation. We calculated the fluxes at $\lambda 5500 \AA$ and $\lambda 6563 \AA$ of a continuum of gas emitted by recombinations and free-free transitions taking into account the temperature in the nebula of AG Dra and found that the flux at $\lambda 5500 \AA$ is greater by $8 \%$ than at $\lambda 6563 \AA$. It was also supposed that the singly ionized helium is dominant. A spherical nebula with a density distribution of $\sim 1 / r^{2}$ in the region $10^{6} \div 10^{10} \mathrm{~cm}^{-3}$, typical of the densities of the symbiotic nebulae, was considered. Carrying out this calculation, we used the mean values for the radius of the cool giant and its mass-loss rate of $30 R_{\odot}$ and $2.3 \times 10^{-7} M_{\odot} \mathrm{yr}^{-1}$ according to the paper of Tomov et al. (2000) and a wind velocity of $30 \mathrm{~km} \mathrm{~s}^{-1}$ (Mikolajewska et al. 1995).

Having the ratio of the fluxes of the nebula at $\lambda 5500 \AA$ and $\lambda 6563 \AA$, we subtracted from the observed $\mathrm{V}$ flux at the epochs of the $\mathrm{H} \alpha$ data the contribution of the giant. The difference is a nebular continuum, which was decreased by $8 \%$ and added to the flux of the giant at $\lambda 6563 \AA$. In this way we obtained the continuum flux of the AG Dra system at this wavelength. For calculation of all line fluxes the photometric data of Skopal (1998), Petrik et al. (1998) and Tomova \& Tomov (1998) were used.
All the fluxes were corrected for an interstellar extinction of $E(B-V)=0.06$ (Mikolajewska et al. 1995; Greiner et al. 1997; Gonzalez-Riestra et al. 1999) using the extinction law of Seaton (1979).

As in our previous work (Tomova \& Tomov 1999), we used the photometric ephemeris $\operatorname{JD}\left(U_{\min }\right)=2442514.4+$ $552.4 \times E$ of Skopal (1994). The zero epoch is that of the photometric minimum, when the cool component is nearer the observer.

\section{The emission measure}

The optical brightenings of AG Dra are caused by an increase in its nebular emission. In Sect. 6 we will consider the possibility that the behaviour of this star during its active 1994-1998 phase can be explained in the framework of one or another model of outburst. Making this analysis we will investigate the changes of the emission measure. Quantitative analysis of the behaviour of the emission measure can be carried out using the Balmer continuum emission, which can be derived from $U$-band photometric data. During the active phase a good sample of optical photometry (Fig. 1) was collected which gives us the possibility to calculate the emission measure at a number of typical epochs of variability of the light. It was already obtained in one of our previous works at some of these epochs (Tomova \& Tomov 1999) but now it is calculated again using another estimate of the distance to the system. We considered the emission measure at all epochs of maxima and minima of the light during 1994-1998 (Table 2). To compare these data with the quiescent ones we took the $U$ fluxes of the circumbinary nebula at the orbital maximum and minimum $F_{\max }^{\text {neb }}=0.175 \times 10^{-12} \mathrm{erg} \mathrm{cm}^{-2} \mathrm{~s}^{-1} \AA^{-1}$ and $F_{\text {min }}^{\text {neb }}=0.040 \times 10^{-12} \mathrm{erg} \mathrm{cm}^{-2} \mathrm{~s}^{-1} \AA^{-1}$, obtained by Tomov et al. (2000). 


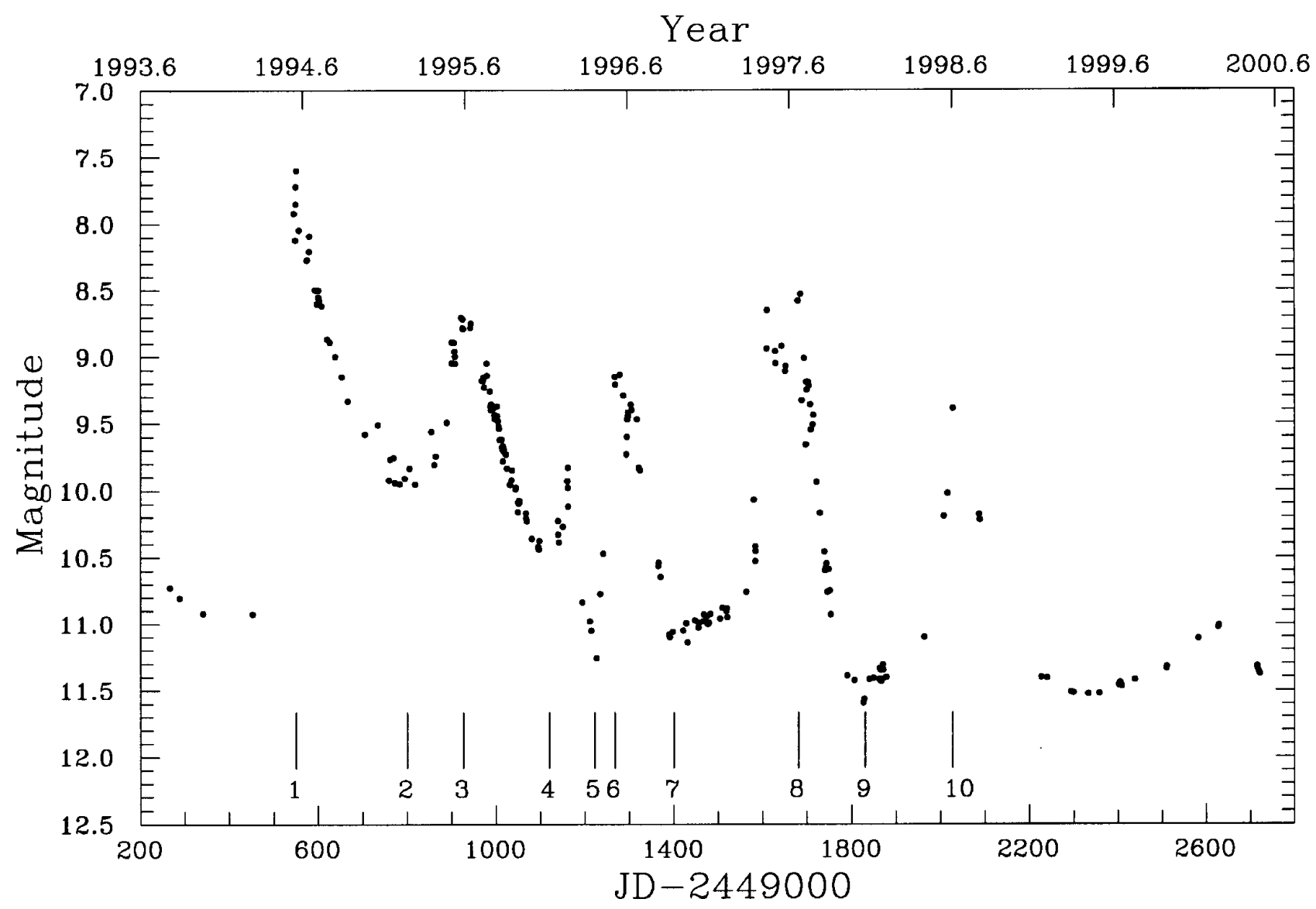

Fig. 1. The $U$ light curve of AG Dra during its active phase based on observations of Skopal et al. (1995), Hric et al. (1996), Montagni et al. (1996), Skopal (1998), Petrik et al. (1998), Tomova \& Tomov (1998) and Tomov et al. (2000). The vertical lines indicate the local extrema under consideration, which are numbered from 1 to 10 and whose times are listed in Table 2.

The magnitudes were converted into continuum fluxes without correcting for the emission lines included in the wavelength region of the $U$ photometric system as we were not provided with spectral data in this region. The fluxes were corrected for the energy distribution of AG Dra. The continuum of this star on the long wavelength-side of the Balmer jump is considerably weaker, which leads to a reduction of the flux at $3650 \AA$. The corrections were made as described in the paper of Tomov et al. (2000). The fluxes were also corrected for the interstellar reddening as described in Sect. 2. Finally we obtained the $U$ fluxes of the circumbinary nebula of AG Dra in its quiescent state (Table 2) subtracting the quiescent fluxes of the stellar components derived in the paper of Tomov et al. (2000). We made also approximate estimates of the fluxes of the hot component for the considered epochs of the active phase using the approach in the same paper and data from the paper of Gonzalez-Riestra et al. (1999). These fluxes were also subtracted from the dereddened fluxes of AG Dra.

To calculate the emission measure we need to know the state of ionization of helium. Mikolajewska et al. (1995) obtained an electron temperature for the nebula of AG Dra of about $15000 \mathrm{~K}$. In our calculations we assumed the same temperature in both the quiescent and the outburst states of the system. The helium ionization state in quiescence was determined in the paper of Tomov et al. (2000) by calculation of the ratio of the emission measures of the neutral and ionized helium. It was allowed that the lines of $\mathrm{He}^{\mathrm{O}}$ are pure recombination lines since the same temperature of $15000 \mathrm{~K}$ was adopted. A ratio $\mathrm{He}^{++} / \mathrm{He}^{+}$ of about 0.5 was obtained, indicating that the singly ionized helium is dominant in the nebula.

During the 1994-1998 stage of activity we found data for some visual lines of $\mathrm{He}^{\mathrm{o}}$ and He II 4686 line at the same epochs at orbital phases, close to the quiescent light maximum in the papers of Gonzalez-Riestra et al. (1999) and Tomova \& Tomov (1999). Only the flux of the narrow emission component of the line He II 4686 was used since its broad component indicates a high velocity stellar wind from the hot companion of the system (see Sect. 5). In this case we obtained a ratio $\mathrm{He}^{++} / \mathrm{He}^{+}$of about 0.4 , which means that the singly ionized helium is dominant in the nebula during outburst too. Such a result was expected since the temperature of the companion was decreased. In our calculations we assumed that the nebular emission is mostly continuum emission of hydrogen and neutral helium. The quantities $\gamma_{\nu}$, related to the emission coefficients of these atoms and determined by recombinations and free-free transitions, were selected as explained 
in the paper of Tomov et al. (2000). A helium abundance of 0.1 (Vogel \& Nussbaumer 1994) was also adopted.

The broad component of the He II 4686 line (Sect. 4) shows a region of high velocity stellar wind present in the nebula of AG Dra during the active phase. The temperature of this region is probably higher than $15000 \mathrm{~K}$. In the paper of Tomova \& Tomov (1999) it was shown that the Balmer continuum emitted by this region is negligible compared to that of the rest of the ionized region(s) of the nebula. That is why we used an electron temperature of $15000 \mathrm{~K}$ when calculating the emission measure.

We adopted a distance of $1.7 \mathrm{kpc}$ as the mean value of the range of distances to the AG Dra system obtained by Tomov et al. (2000). So for the quiescent emission measures we derived $n_{\mathrm{e}}^{2} V=1.59 \times$ $10^{59}(\mathrm{~d} / 1.7 \mathrm{kpc})^{2} \mathrm{~cm}^{-3}$ at the orbital maximum and $n_{\mathrm{e}}^{2} V=0.36 \times 10^{59}(\mathrm{~d} / 1.7 \mathrm{kpc})^{2} \mathrm{~cm}^{-3}$ at the orbital minimum.

The results of the calculations of the data taken during the active phase are presented in Table 2. The observed $U$ magnitude is listed in the fifth column, the flux of the nebula in the sixth column and its emission measure in the seventh column. The ratio $m$ of the emission measure at some epoch during the active phase and the emission measure at the quiescent orbital maximum is listed in the eighth column. The data show that during the active phase the emission measure changes by factors from 0.5 to 36 compared to its maximal value at the quiescent state. The phase of the light minimum after the 1997 outburst is very close to the orbital minimum, but the emission measure is by a factor of about 2 greater than this one at the quiescent orbital minimum, indicating greater number of recombinating ions. In our opinion this is most probably a result of the increased loss of mass of the two stellar components during the outburst (see Sect. 6).

\section{The $\mathrm{H} \alpha$ line}

The $\mathrm{H} \alpha$ line of AG Dra was analysed using spectral data obtained by us at the end of the quiescent stage before the last active phase as well as immediately before and after the epoch of the 1994 light maximum (Tomova \& Tomov 1999). In the present work we continue our analysis using data derived during the 1996 and 1997 brightenings and the lower state of the light between them (Table 1). All of our data reveal a single-peaked profile of the line (Fig. 2) with unusually extended low intensity wings, reaching the level of the continuum at a distance not less than $2000 \mathrm{~km} \mathrm{~s}^{-1}$ from its center during all the time of observation. The wings were always symmetrical but an asymmetry in the upper part of the line was always present. This asymmetric part was confined by a small shoulder that was hardly visible and was located below the level of the half maximum on the short wavelengthside. Since the wings were symmetrical and the feature causing the asymmetry was related to the upper part of the line, being based on our previous analysis (Tomova \& Tomov 1999), we conclude that this feature is probably

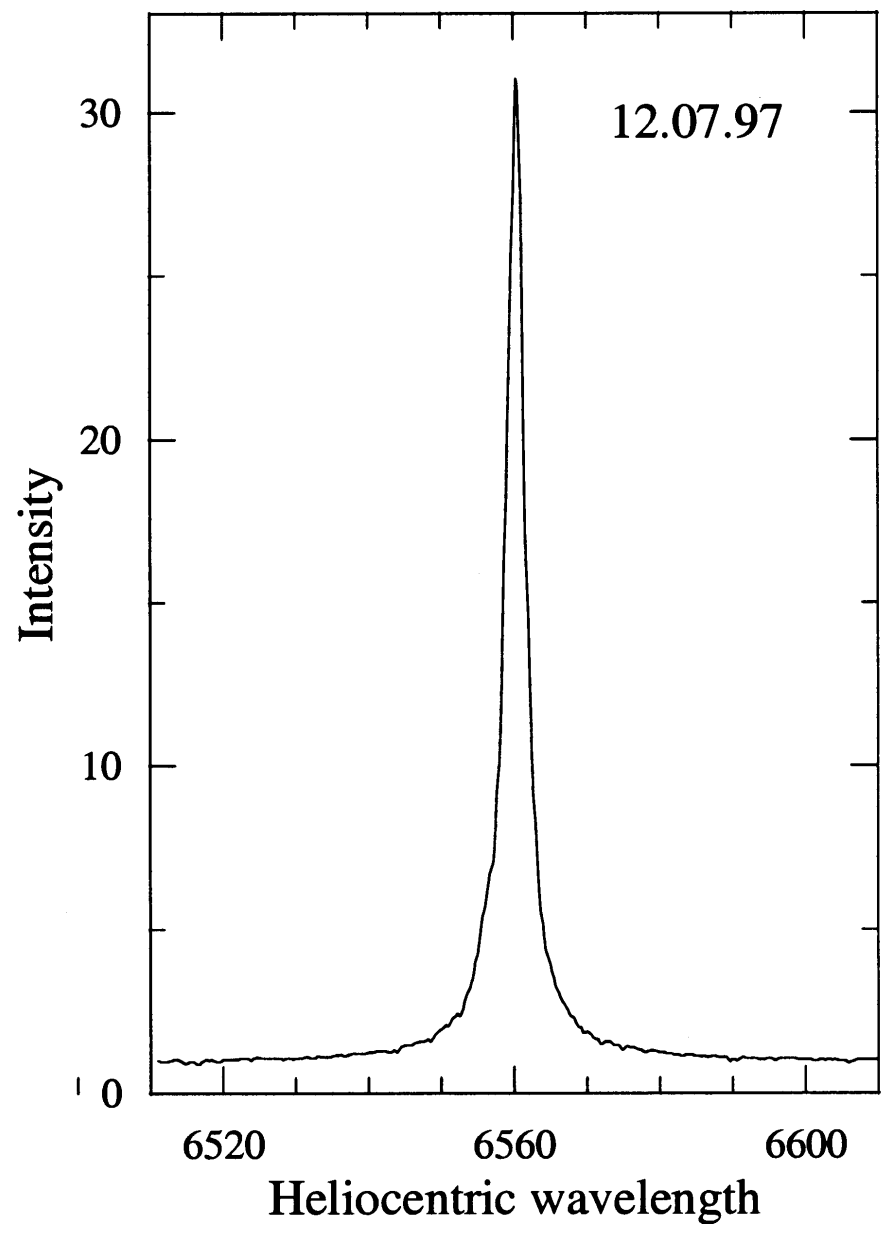

Fig. 2. $\mathrm{H} \alpha$ profile of AG Dra normalized with respect to the local continuum.

determined by absorption of some part of the $\mathrm{H} \alpha$ photons. The profile varied as a result of changes in this feature during the time of our observations, which from its side was probably determined by changes of the optical depth.

The intensity of the wings also changed in correlation with the optical light (Fig. 1). It was higher during the times of the two brightenings of the star compared with its value in the lower state of the light between them (Fig. 3). The behaviour of the wings shows that greater numbers of emitting hydrogen atoms were observed at the times of the increased light compared with its lower value. We suppose (Sect. 6) that the increased number of emitting $\mathrm{H} \alpha$ line atoms in the circumbinary nebula of the AG Dra system during its active 1994-1998 phase is probably determined by an increase of both the mass-loss rate of its cool giant and the Lyman photon luminosity of the hot companion.

The changes of the width $(F W H M)$ of the line were also in correlation with the light. During the times of the optical outbursts it was greater, reaching values of about $160 \mathrm{~km} \mathrm{~s}^{-1}$ at the 1996 light maximum and about $140-150 \mathrm{~km} \mathrm{~s}^{-1}$ at the 1997 one. During the lower state of the light it was about $110 \mathrm{~km} \mathrm{~s}^{-1}$. The data indicate a more pronounced turbulence of the nebular environment during the times of light increase. 

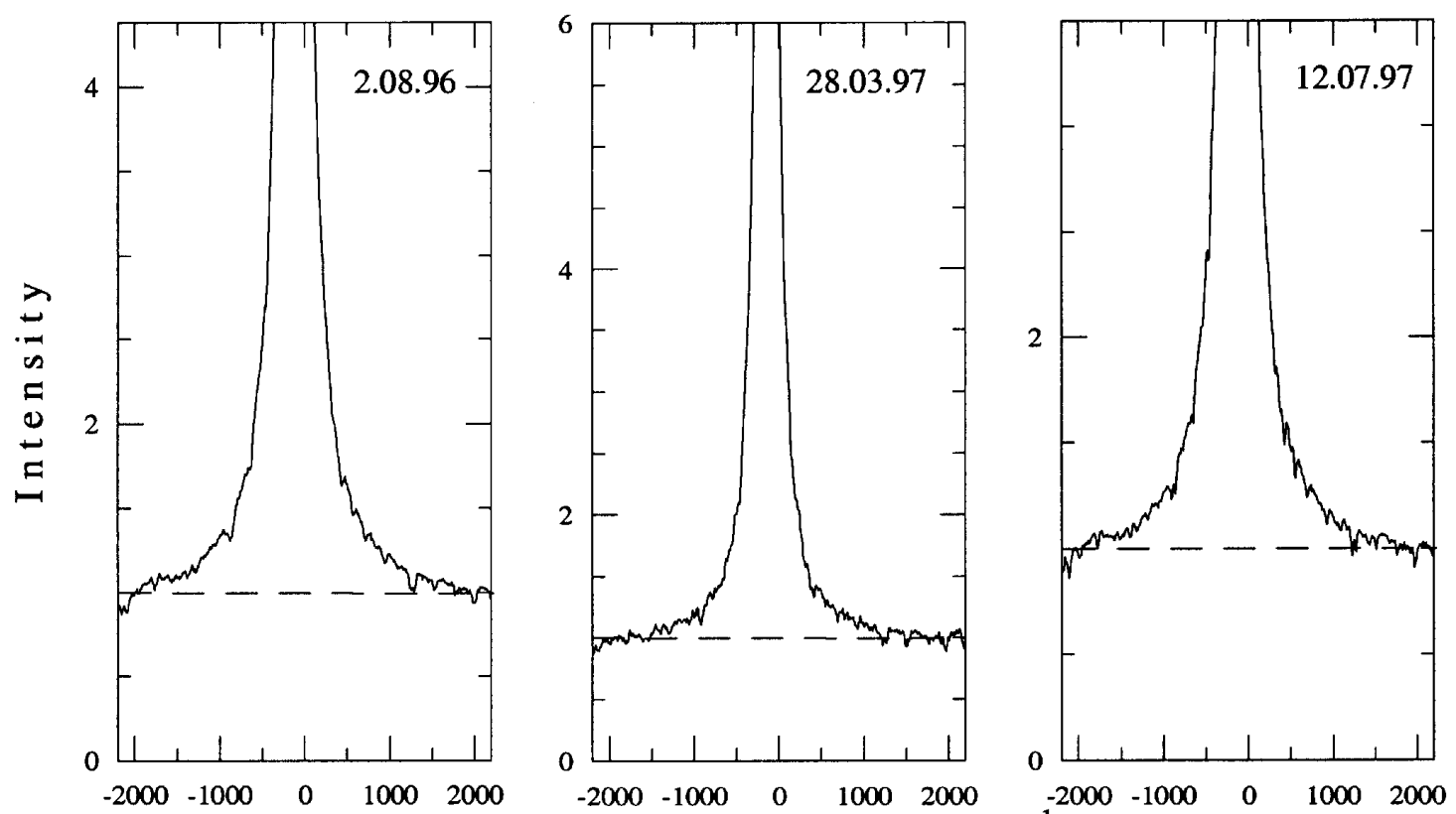

Heliocentric R V $\left(\mathrm{km}^{\left.-\mathrm{s}^{-1}\right)}\right.$

Fig. 3. Variations of the wings intensity of the $\mathrm{H} \alpha$ line. For a direct comparison the ratio of the ordinate scales was chosen to be equal to the ratio of the continuum fluxes at the position of $\mathrm{H} \alpha$.

Table 3. The $\mathrm{H} \alpha$ line data.

\begin{tabular}{llll}
\hline \hline JD & Phase & $\begin{array}{l}R V \\
\mathrm{~km} \mathrm{~s}^{-1}\end{array}$ & \\
\hline $2450000+$ & & -129.41 & 136.203 \\
\hline 293.321 & 0.082 & -125.98 & 148.174 \\
295.489 & 0.086 & -125.22 & 146.124 \\
298.396 & 0.091 & -150.06 & 126.058 \\
321.383 & 0.133 & -153.90 & 83.090 \\
475.523 & 0.412 & -142.80 & 85.192 \\
536.333 & 0.522 & -138.32 & 82.028 \\
565.383 & 0.574 & -136.49 & 137.474 \\
618.419 & 0.670 & -138.91 & 154.769 \\
641.515 & 0.712 & -146.27 & 175.268 \\
674.340 & 0.772 & -142.20 & 202.230 \\
703.243 & 0.824 & -142.62 & 204.238 \\
705.332 & 0.828 & -151.37 & 173.298 \\
732.356 & 0.877 & & \\
\hline
\end{tabular}

${ }^{a}$ Emission line flux in units $10^{-12} \mathrm{erg} \mathrm{cm}^{-2} \mathrm{~s}^{-1}$.

The behaviour of the $\mathrm{H} \alpha$ flux is shown in Fig. 4 and its data are listed in Table 3 . It also varies with the light. The wavelength position of the line was measured in the area of the wings as its other part was influenced by selfabsorption (Table 3 ). Its radial velocity shows pronounced variation with the orbital phase during this stage of activity (Fig. 5). In our view these data can be analysed only

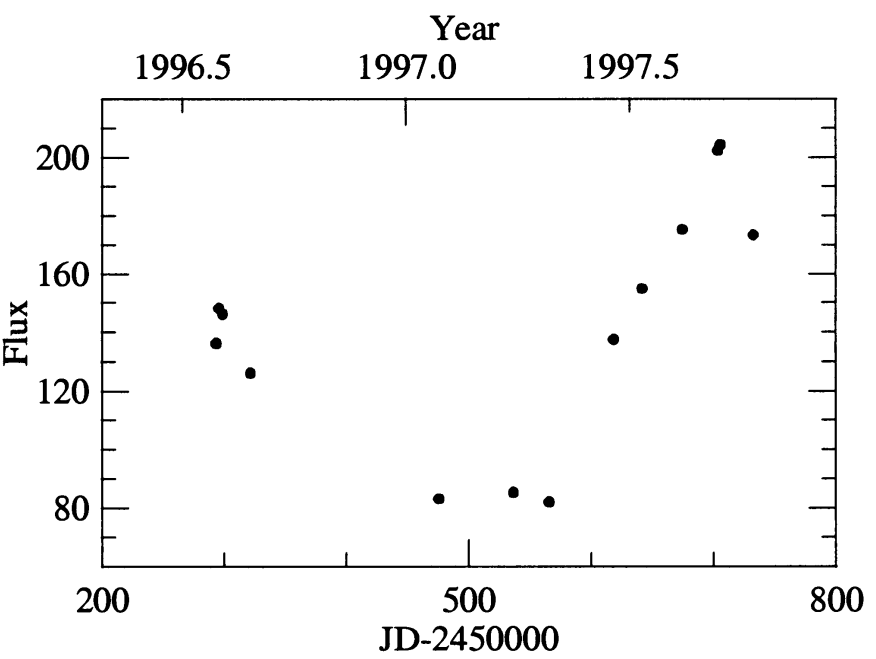

Fig. 4. H $\alpha$ flux in units $10^{-12} \mathrm{erg} \mathrm{cm}^{-2} \mathrm{~s}^{-1}$ during the time of observation.

when the quiescent dynamical behaviour of the circumbinary nebula is known.

\section{The helium lines}

\subsection{The Hel 4713 line}

During the time of all observations it had a nebular emission profile and a width $(F W H M)$ of about $70 \mathrm{kms}^{-1}$. The data of its flux and radial velocity are listed in Table 4 . The radial velocity showed variation with the orbital phase. As in the case of the $\mathrm{H} \alpha$ line we will consider this variation only when the results of an analysis of its quiescent data are available. 


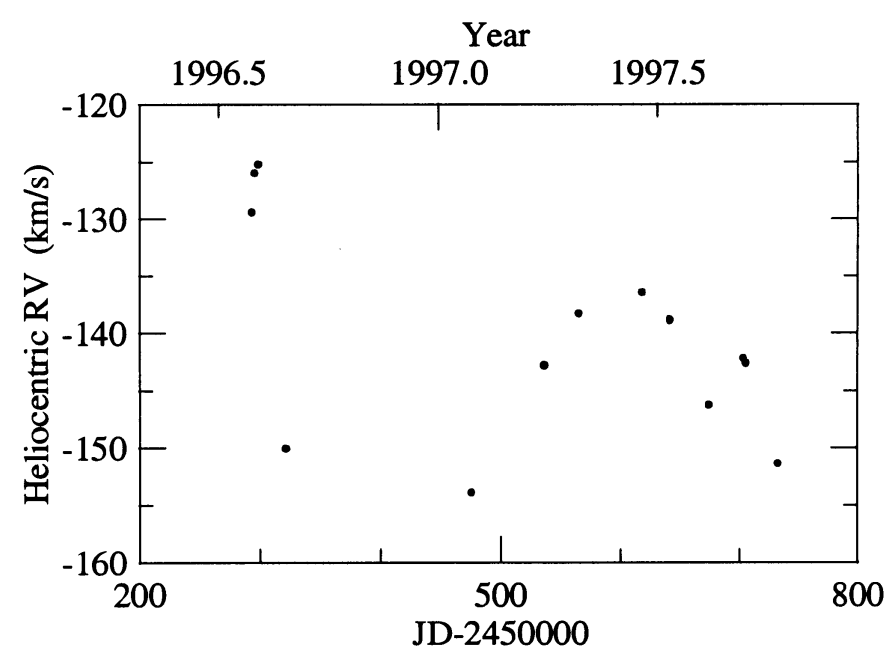

Fig. 5. $\mathrm{H} \alpha$ radial velocity during the time of observation.

Table 4. The He I 4713 line data.

\begin{tabular}{llll}
\hline \hline $\begin{array}{l}\text { JD } \\
2450000+\end{array}$ & Phase & $\begin{array}{l}R V \\
\mathrm{~km} \mathrm{~s}^{-1}\end{array}$ & $\begin{array}{l}F \times 10^{-12} \\
\mathrm{erg} \mathrm{cm}{ }^{-2} \mathrm{~s}^{-1}\end{array}$ \\
\hline 293.394 & 0.082 & -134.785 & 0.926 \\
322.282 & 0.134 & -164.299 & 0.565 \\
677.283 & 0.777 & -146.807 & 2.158 \\
703.307 & 0.824 & -150.814 & 1.561 \\
732.274 & 0.877 & -167.798 & 0.651 \\
\hline
\end{tabular}

\subsection{The Hell 4686 line}

In July and August 1996 as well as during JuneSeptember 1997 the He II 4686 line consisted of two emission components - a central narrow component with width $(F W H M)$ equal to about $90 \mathrm{kms}^{-1}$ and a broad one whose width was much greater (Fig. 6). Only the last spectrum, taken in October 1997, revealed one component profile of this line when only a narrow emission component was present. We measured the intensity of the broad component in the following way. The observed spectrum in its region was corrected through removing some weak emission lines of $\mathrm{O}$ II and NII as well as the strongest absorption lines of the giant. Then it was analysed by fitting with Gaussian function. The parameters obtained with this procedure are listed in Table 5. The equivalent width and the flux of the line were derived with an error ranging from $10 \%$ to $26 \%$ depending on the error of the local continuum of the individual spectra. The wide of the line at the continuum level $(F W Z I)$ was considered by us to be determined from that distance from the line center where the fit reaches the level of the noise. The velocity of the stellar wind (see below) of the hot companion $v_{\text {hot }}$ (Table 5 ) is equal to the half of the $F W Z I$ of the line. The values of this velocity in the table were determined taking also into account the error of the line width $(F W H M)$. It was not possible to fit the broad

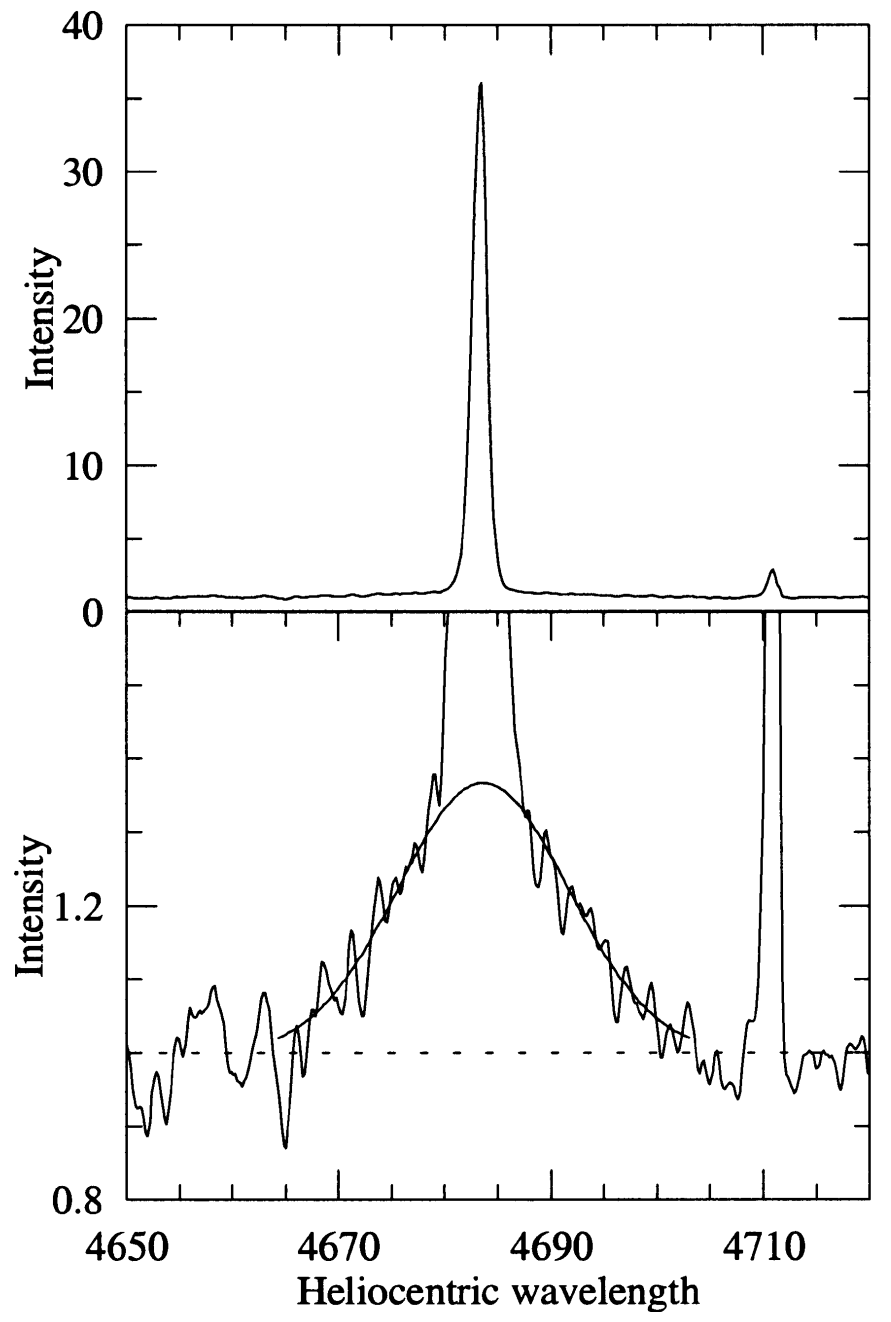

Fig. 6. Upper panel: the profile of the He II 4686 line, based on a CCD frame on 16.08.97. Lower panel: the area of its wings where the broad component is seen. The level of the local continuum is marked with a dashed line.

component with good accuracy on the spectrum taken on 19 June 1997 (JD 2450619 ) because of cosmic rays on its red wing. However taking into account the data based on the other spectra, we consider that the hot companion of the system has also a stellar wind with a velocity of about $1000 \mathrm{~km} \mathrm{~s}^{-1}$ and a mass-loss rate of $\sim 10^{-7} M_{\odot} \mathrm{yr}^{-1}$ at that time.

In our previous work (Tomova \& Tomov 1999) we considered different possible mechanisms of line broadening of the Balmer lines of AG Dra and came to the conclusion that the broad component of $\mathrm{H} \gamma$ is mainly due to a stellar wind with a velocity of $800 \mathrm{~km} \mathrm{~s}^{-1}$, being at the same time affected by electron scattering. Now we will check if the broad component of the He II 4686 line is due to the same mechanisms.

We first consider the possibility that this component is determined only by electron scattering. The total flux of the line, which is a sum of the fluxes of the two components ranges from $23.218 \times 10^{-12} \mathrm{erg} \mathrm{cm}^{-2} \mathrm{~s}^{-1}$ to $49.548 \times$ $10^{-12} \mathrm{erg} \mathrm{cm}^{-2} \mathrm{~s}^{-1}$ (Table 5). With the parameters of 
Table 5. The He II 4686 line data.

\begin{tabular}{|c|c|c|c|c|c|c|c|c|}
\hline $\begin{array}{l}\text { JD } \\
2450000+\end{array}$ & Phase & $\begin{array}{l}R V(\mathrm{~N}) \\
\mathrm{km} \mathrm{s}^{-1}\end{array}$ & $\begin{array}{l}F(\mathrm{~N}) \times 10^{-12} \\
\mathrm{erg} \mathrm{cm}^{-2} \mathrm{~s}^{-1}\end{array}$ & $\begin{array}{l}F W H M(\mathrm{~B}) \\
\mathrm{km} \mathrm{s}^{-1}\end{array}$ & $\begin{array}{l}F W Z I(\mathrm{~B}) \\
\mathrm{km} \mathrm{s}^{-1}\end{array}$ & $\begin{array}{l}v_{\text {hot }} \\
\mathrm{kms}^{-1}\end{array}$ & $\begin{array}{l}F(\mathrm{~B}) \times 10^{-12} \\
\mathrm{erg} \mathrm{cm}^{-2} \mathrm{~s}^{-1}\end{array}$ & $\dot{M}_{\mathrm{hot}}$ \\
\hline 293.394 & 0.082 & -139.67 & 28.318 & $1223 \pm 82$ & 2404 & 1200 & 3.221 & 1.18 \\
\hline 322.282 & 0.134 & -157.54 & 20.722 & $1188 \pm 154$ & 2212 & 1100 & 2.496 & 0.96 \\
\hline 619.369 & 0.672 & -167.72 & 33.273 & & & & & \\
\hline 677.283 & 0.777 & -154.19 & 42.559 & $1213 \pm 56$ & 2556 & 1300 & 6.989 & 1.89 \\
\hline 703.307 & 0.824 & -149.74 & 42.444 & $1055 \pm 58$ & 2252 & 1100 & 5.147 & 1.38 \\
\hline 732.274 & 0.877 & -161.65 & 25.900 & & & & & \\
\hline
\end{tabular}

$\mathrm{N}$ - narrow component.

B - broad component.

$\dot{M}_{\text {hot }}=\dot{M}_{\text {hot }} \times 10^{-7}(\mathrm{~d} / 1.7 \mathrm{kpc})^{3 / 2} M_{\odot} \mathrm{yr}^{-1}$.

the nebula adopted in Sect. 3 this gives emission measures of $0.59 \div 1.25 \times 10^{59}(\mathrm{~d} / 1.7 \mathrm{kpc})^{2} \mathrm{~cm}^{-3}$. Assuming a constant electron density of $10^{10} \mathrm{~cm}^{-3}$ (Mikolajewska et al. 1995) we obtain a radius of $5.19 \div$ $6.69 \times 10^{12}(\mathrm{~d} / 1.7 \mathrm{kpc})^{2 / 3} \mathrm{~cm}$ of the spherical emitting volumes. If the broad component was produced only by electron scattering it would appear in regions with optical thickness of about $0.11 \div 0.15$. Using these optical thicknesses and an electron density of $10^{10} \mathrm{~cm}^{-3}$, we obtain $1.71 \div 2.29 \times 10^{13}(\mathrm{~d} / 1.7 \mathrm{kpc})^{2 / 3} \mathrm{~cm}$ for the radius of these regions, corresponding to enormous emission measures of $2.094 \div 5.004 \times 10^{60}(\mathrm{~d} / 1.7 \mathrm{kpc})^{2} \mathrm{~cm}^{-3}$. Since this result is in disagreement with the previous one, we conclude that the broad component is probably not produced only by electron scattering.

This point would be more clear if we analyzed the data of Gonzalez-Riestra et al. (1999) for this line as well. They reveal also the presence of two emission components and were obtained at two stages of the evolution of the system - the quiescent one before 1994 and the active phase after this time. The broad emission component was absent at some epochs during quiescence, for instance on 10 Jan. 1993. On 5 Jan. 1994, however, it was present and if supposed to be due to electron scattering, the optical thickness of its emitting region will be equal to 0.08 . In this case the radius and the emission measure are found to have values close to those ones calculated with the total flux of the line and our assumption of electron scattering only cannot be rejected. A broad component was observed on 19 Aug. 1995 too and when supposed to be a result of electron scattering the optical thickness is derived to be 0.14. In this case the radius of the emitting region and its emission measure are found to be considerably greater than those determined by the total line flux. This means that the broad component was not due only to electron scattering. Thus, when the intensity of the broad component of the He II 4686 line is low it can be due only to electron scattering. However at the times July-August 1996 and June-September 1997 another process contributes to its appearance.
An emission line similar to the He II 4686 broad component can be radiated by a small accretion disk around the compact object, rotating with Keplerian velocity. Let us consider whether this was the case in July-August 1996 and June-September 1997 when the intensity of this component was high. For the radius of the secondary component of the system we adopt a value of $0.10 R_{\odot}$ (see below). The mass of this component was assumed by Mikolajewska et al. (1995) to be about $0.6 M_{\odot}$ and by Gonzalez-Riestra et al. (1999) to be about $0.3 M_{\odot}$. We suppose it is in the range $0.3 \div 0.6 M_{\odot}$ and will perform our calculations with these two borderline values. A Keplerian velocity of $757 \div 1070 \mathrm{~km} \mathrm{~s}^{-1}$ is obtained, which is comparable to the velocity of about $1100 \div 1300 \mathrm{~km} \mathrm{~s}^{-1}$, corresponding to the $F W Z I$ of the broad component (Table 5). A small disk rotating with this velocity and located close to the hot secondary would appear as a result of accretion of a stellar wind and its luminosity would be low. In our previous work (Tomova \& Tomov 1999) we came to the conclusion that the existence of such a disk is not plausible either in the case when the outbursts are caused by thermonuclear events or when they are driven by accretion. At present we will consider the possibility for it to exist in the case when the activity of AG Dra is assumed to take place in the framework of the scenario proposed for its X-ray emission (Greiner et al. 1997). According to the optical spectral data (Gonzalez-Riestra et al. 1999; Tomova \& Tomov 1999; this work) its presence can be supposed only at the times of the decrease of the light after its maxima. If the activity is determined by this mechanism the existence of a disk will be doubtful, since accretion is probably not realized at the times of the light decrease because of the appearance of a stellar wind from the expanding photosphere of the compact object. But if we suppose that the expansion decreases only because of the reduction of the accretion rate to its quiescent value the disk would be more luminous before the light maximum, but not after it, which does not explain the observed behaviour of the broad component of the He II 4686 line. Thus, we will assume that this component is mainly caused by high 
velocity stellar wind, appearing most probably as a result of the outbursts themselves.

A high velocity wind, however, can be generated in two cases - by a hot stellar object and an accretion disk. The possibility for an origin from a disk was considered in the work of Tomova \& Tomov (1999), where it was stated that observational data supporting the existence of an accretion disk in the AG Dra system were not available till that time. Thus, we conclude that the broad components of the optical emission lines of this system at the times of activity after its light maxima are probably due to wind of a hot compact object rather than to an accretion disk. However, the wind velocity, which we obtained, can be considered as an upper limit since these components are also affected by electron scattering.

To determine the mass-loss rate of the hot compact secondary of the AG Dra system we applied the nebular approach, as in the work of Vogel \& Nussbaumer (1994), based on the relation of this parameter with the line flux when the wind has spherical symmetry and a constant velocity. Vogel \& Nussbaumer (1994) used the flux of the He II 1640 line, supposed to be dominated by recombination. They noted that the created photons are predominantly scattered and not destroyed, escaping as a result from the wind. That is why we used the approach of these authors, assuming that if the He II 4686 line is actually not optically thin, it will be scattered too.

The particle density in the wind is a function of the distance to the center and is expressed via the continuity equation. The electron temperature was adopted to be $T_{\mathrm{e}}=20000 \mathrm{~K}$ and the state of the helium ionisation was supposed to be $\mathrm{He}^{++}$as in the work of Tomova \& Tomov (1999). We also used a recombination coefficient of $2.689 \times 10^{-13} \mathrm{~cm}^{3} \mathrm{~s}^{-1}$ for case $\mathrm{B}$, corresponding to this temperature and a density of $10^{13} \mathrm{~cm}^{-3}$ (Storey \& Hummer 1995) and a parameter $\mu$ of 1.4 (Nussbaumer \& Vogel 1987) determining the mean molecular weight $\mu \mathrm{m}_{\mathrm{H}}$ in the hot wind. As the line flux is thought to be emitted by a spherical region, the radii of integration must be determined. Since we treat the wind in the nebular approach, the inner radius is thought to be the radius of the star. We used the value of this parameter based on the analysis of Greiner et al. (1997). An appearance of a stellar wind from the compact object is considered by these authors to be possible when its photosphere has expanded as a result of the increasing accretion rate. In this case the radius is assumed to be about $0.14 R_{\odot}$ at a distance to the system of $2.5 \mathrm{kpc}$. Since we used a distance of $1.7 \mathrm{kpc}$ the radius was reduced to $0.10 R_{\odot}$. In our view it would not be correct to use the data for the radius during outburst of Mikolajewska et al. (1995) and Gonzalez-Riestra et al. (1999) since these data are related to the case of an increase of the bolometric luminosity, and according to the scenario of Greiner et al. (1997) it remains invariable. Let now determine the outer radius of integration. The numerical calculations of the evolution of the nebular environment in symbiotic systems (Nussbaumer \& Walder 1993) show that six months after the appearance of the wind of the hot secondary its region has a size comparable with the binary separation. We could not determine this region during the last active phase of AG Dra as the variation of the mass-loss rate of its cool giant is not known. On the other hand it is easy to show that regions of high velocity wind far from its base have a negligible contribution. Thus, we will use an outer radius of integration equal to half of the binary separation, $166 R_{\odot}$. Before considering our calculation of the mass-loss rate based on the broad component of the He II 4868 line we will calculate this parameter using the broad component of the $\mathrm{H} \gamma$ line from the work of Tomova \& Tomov (1999), as a distance to the system of $1.7 \mathrm{kpc}$ is now adopted. This component, having a flux of $2.38 \times 10^{-12} \mathrm{erg} \mathrm{cm}^{-2} \mathrm{~s}^{-1}$, was observed after the 1995 light maximum and was supposed to be due mainly to stellar wind, whose velocity was determined to be less than $800 \mathrm{~km} \mathrm{~s}^{-1}$. A recombination coefficient of $1.233 \times 10^{-14} \mathrm{~cm}^{3} \mathrm{~s}^{-1}$ for the same density and temperature was adopted (Storey \& Hummer 1995). The mass-loss rate in this case amounts to less than $0.97 \times$ $10^{-7}(\mathrm{~d} / 1.7 \mathrm{kpc})^{3 / 2} M_{\odot} \mathrm{yr}^{-1}$. The mass-loss rates based on the current data (Table 5) can be also regarded as upper limits because of the electron scattering contribution in the broadening of the line.

\section{Discussion}

The active 1994-1998 phase of the symbiotic star AG Dra is characterized by several increases of the optical light and the amplitude of the greater of them was more than three magnitudes in the $U$-band photometric system. Broad emission components indicating mainly high velocity stellar wind from the compact companion are present in the spectrum after the 1995 (Tomova \& Tomov 1999), 1996 and 1997 light maxima. According to the evolutionary models of an accreting white dwarf (Shara et al. 1993; Kato \& Hachisu 1994; Yungelson et al. 1995) the total mass lost by it is approximately equal to the mass of the accumulated hydrogen envelope. If we suppose that the whole envelope has been lost during the time of light decrease, the accretion must be realized at a rate close to that of the loss of mass $10^{-8} \div 10^{-7} M_{\odot} \mathrm{yr}^{-1}$, since these two processes had approximately the same duration. However, when the accretion rate is $10^{-8} \div 10^{-7} M_{\odot} \mathrm{yr}^{-1}$ a steady burning of hydrogen is realized which excludes the possibility of a thermonuclear event (Mikolajewska \& Kenyon 1992). Consequently the optical brightenings of AG Dra after 1994 most probably are not determined by thermonuclear events at the surface of a white dwarf.

For an explanation of the X-ray spectrum, Greiner et al. (1997) proposed a model where the accretion rate is increased, leading to an expansion of the compact secondary at constant bolometric luminosity. The growth of the $U$ light, however, is caused by an enhancement of the nebular continuum, which is determined by increase in the number of the recombining ions. During the 1994 and 1995 light maxima the $U$ flux of the circumbinary nebula and its emission measure increased by factors of 36.2 and 
12.9 compared to their quiescent maximal values. At 1996, 1997 and 1998 light maxima they increased by factors of $8.3,14.9$ and 6.6 respectively. The intensity of the nebular continuum is proportional to the ion density. There is also a linear dependence between the ion density and the density of the radiation field of the ionizing star. Then if the compact companion is supposed to be this source, which heats the circumbinary nebula, which from its side is partly ionized, the companion's Lyman photon luminosity must increase proportionally to the nebular $U$ flux and the emission measure. Supposing a black body energy distribution and using a distance to the system of $1.7 \mathrm{kpc}$, the data of Greiner et al. (1997) for the luminosity and the temperature of the hot component as well as the velocity of its expansion calculated by them, we find that the Lyman photon luminosity of this star increases by a factor of about 3. Our supposition for a black body was based on the UV data analysis of Gonzalez-Riestra et al. (1999) as well as on the generally accepted view for the continuum energy distribution of the hot stellar components of the symbiotic binaries (Mürset et al. 1991), although they emit mostly in the far ultraviolet - a region not entirely observed by current instrumentation. The increase of the Lyman luminosity by a factor of 3 is too small compared with the growth of the $U$ flux and the emission measure and an additional ionization mechanism is need.

Let us consider the increase of the amount of the emitting gas following the scenario proposed by Greiner et al. (1997), since an appearance of a stellar wind from the compact companion, such as probably observed, is supposed in the framework of this scenario. Friedjung et al. (1998) came to the conclusion that the variation of the quiescent $U$-band flux of AG Dra at the time of its orbital maximum is caused by changes in its cool giant's wind. Later, the quiescent $U$ orbital modulation of this system was considered by Tomov et al. (2000). They inferred that the variation of the maximum $U$ flux will be determined by the giant's mass-loss rate only when the hot companion of the system has an excess of luminosity, which makes it able to ionize practically the whole circumbinary nebula, except the region behind the giant. Consequently, the growth of the companion's luminosity by itself will not lead to an increase in the number of ions recombining in the nebula during the active phase. This can be also caused by an increase in the mass-loss rate of the giant star. Such a mechanism was initially suggested by Nussbaumer \& Vogel (1988) to explain the optical outbursts of symbiotic stars. Moreover the analysis of the radial velocities by Galis et al. (1999) suggests that the cool giant pulsates, which probably leads to changes in its mass-loss rate.

The strongest of the outbursts of AG Dra during its last active phase was the 1994 one, when the nebular $U$ flux increased by a factor of 36.2 . It requires a growth of the mass-loss rate by a factor of 6 if the radius of the star does not change. At the same time, however, to produce a hydrogen-rich envelope with a mass of $\geqq 5 \times 10^{-5} M_{\odot}$ onto a white dwarf with mass less than $0.6 M_{\odot}$ in a time of $100^{\mathrm{d}}$, that is supposed to be the typical time of the $\mathrm{X}$-ray flux decrease, the mass-loss rate of the giant must increase several orders of magnitude above its quiescent value (Greiner et al. 1997). This is an important difficulty met by this model. Its solving is a problem of a theoretical nature and is not included in our consideration.

We propose the following sequence when an outburst takes place:

1. The $U$ light begins to increase because of the appearance of a shell in the wind of the giant and rises while the hot companion is able to maintain ionization in the same region of the nebula as in quiescence;

2. When the ionized region begins to shrink as a result of the increase of the mass-loss, the light stops its rise and remains constant, while the shell reaches the companion and its accretion rate begins to increase;

3. The increased accretion rate leads to the creation of an envelope and gives rise to the Lyman luminosity, the companion ionizes an additional part of the nebula and the light rises for a longer time. It reaches a maximal value together with the Lyman luminosity when a high velocity wind appears. So the time of rise of the light is more than $80^{\mathrm{d}}-90^{\mathrm{d}}$, the time needed for a particle of the giant's wind to travel the distance between the two stars. The wind velocity is not supposed to change during the active phase and remains approximately the same, $30 \mathrm{~km} \mathrm{~s}^{-1}$ (Mikolajewska et al. 1995). The massloss rate of the giant declines to its quiescent value before the epoch of the light maximum;

4. The wind of the companion stops the accretion and contributes to the expansion of the ejected shell. At the same time the accreted envelope burns out and the Lyman luminosity decreases to its quiescent value. The two processes determine the return of the $\mathrm{U}$ light to its quiescent level.

There is a problem related to the mass of the accreted envelope. Neither the mass-loss at a rate of $\sim 10^{-7} M_{\odot} \mathrm{yr}^{-1}$ nor the burning of hydrogen-rich material at a rate of $\sim 10^{-8} M_{\odot} \mathrm{yr}^{-1}$, such as that proposed by Greiner et al. (1997), can use up an envelope with a mass of $\sim 10^{-5} M_{\odot}$ and cause the contraction of the white dwarf back to its original state for the typical time of the X-ray flux increase to its quiescent value.

This scenario gives, though, a possibility to explain some details of the curve (Fig. 1) - for example, that the light after its 1994 maximum dropped to a level considerably higher than its quiescent value. The reason can be an early increase of the mass-loss rate of the giant. On the other hand, however, the analysis of same observational data does not confirm our supposition that most of the circumbinary nebula is an ionized region during quiescence, with the exeption of the region behind the giant. For instance Schmid et al. (1999) obtained high efficiency for the scattering of radiation in the O VI doublet $1032-1038$ by neutral hydrogen in the atmosphere of the giant of AG Dra at an orbital phase close to the spectral quadrature and supposed the presence of a dense neutral 
region. However, these authors note that such a dense region would also produce a strong Rayleigh scattering of the continuum radiation, which has never been detected by IUE.

\section{Conclusions}

We present results of $U$ photometric observations as well as CCD spectral observations carried out in the regions of the $\mathrm{H} \alpha$ and He II 4868 lines of the spectrum of the symbiotic binary AG Dra. The photometric data are related to the whole 1994-1998 active phase of this system and the spectral data only to its 1996 and 1997 outbursts. We derived profiles, fluxes and radial velocity data of the lines H $\alpha$, He I 4713 and He II 4868.

The photometric data give us the possibility to obtain the emission measure of the circumbinary nebula adopting a distance to the system of $1.7 \mathrm{kpc}$ and using only the nebular contribution to the observed $U$ flux. Emission measures of $n_{\mathrm{e}}^{2} V=1.59 \times 10^{59}(\mathrm{~d} / 1.7 \mathrm{kpc})^{2} \mathrm{~cm}^{-3}$ and $n_{\mathrm{e}}^{2} V=0.36 \times 10^{59}(\mathrm{~d} / 1.7 \mathrm{kpc})^{2} \mathrm{~cm}^{-3}$ at the orbital photometric maximum and minimum were obtained on the basis of the quiescent $U$ flux. At the times of the five maxima of the $U$ light during the 1994-1998 active phase the emission measure was increased by factors of 36,13 , 8, 15 and 7 compared with its quiescent maximal value.

Variations of the width $(F W H M)$, the flux and the intensity of the wings of the line $\mathrm{H} \alpha$ were observed during the time 1996-1997 correlating with the light. The behaviour of the wings showed an increase of the number of emitting atoms in the nebula during the outbursts in agreement with the increase of the emission measure. The FWHM showed a more pronounced gas turbulence during the times of activity.

After the visual light maxima in 1996 and 1997 the profile of the He II 4868 line consisted of two emission components: a central narrow component and a broad component indicating mainly a stellar wind. This wind had a velocity of $1100 \div 1300 \mathrm{~km} \mathrm{~s}^{-1}$ and was probably produced by the compact companion, which was supposed to lose mass at a rate of about $1 \div 2 \times 10^{-7}(\mathrm{~d} / 1.7 \mathrm{kpc})^{3 / 2} M_{\odot} \mathrm{yr}^{-1}$. A loss of mass of AG Dra via a high velocity wind was observed during its 1995 brightening, too (Tomova \& Tomov 1999). Based on these data we conclude that the outbursts of this system during its 1994-1998 active phase most probably are not thermonuclear, since the accretion rate in this case must be close to this one of the loss of mass. The mass-loss rate, obtained by observation, has a high value and accretion at a rate close to it falls into the region of a steady-state burning of hydrogen at the surface of a white dwarf.

The growth of the Lyman photon luminosity of the companion is less than the growth of the emission measure of the nebula and is not sufficient to explain the optical outbursts that are caused by variation of the nebular emission. Using one of the conclusions of the work of Tomov et al. (2000) that the luminosity of the companion is high, providing the possibility that it ionizes practically the whole surrounding nebula except for the region behind the giant, we assume that the optical outbursts of the system could be partly due to a growth of the massloss rate of the giant and could be also interpreted in the framework of the model proposed for its X-ray emission (Greiner et al. 1997). According to this idea the optical outbursts of AG Dra would be the result of both an increase in the mass-loss rate of its cool giant and an increase of the Lyman photon luminosity of the hot companion.

Acknowledgements. The authors thank their colleague Dr. R. K. Zamanov for providing part of the CCD spectra. They are also grateful to the referee Dr. M. Friedjung whose remarks contributed to improvement of the paper and to Dr. N. Markova for the helpful discussion.

\section{References}

Friedjung, M., Hric, L., Petrik, K., \& Galis, R. 1998, A\&A, 335,545

Galis, R., Hric, L., Friedjung, M., \& Petrik, K. 1999, A\&A, 348,533

Gonzalez-Riestra, R., Viotti, R., Iijima, T., \& Greiner J. 1999, A\&A, 347, 478

Greiner, J., Bickert, K., Luthardt, R., et al. 1997, A\&A, 322, 576

Griffin, R. E. M., \& Lynas-Gray, A. E. 1999, AJ, 117, 2998

Hric, L., Skopal, A., Urban, Z., et al. 1996, Contrib. Astron. Obs. Skalnaté Pleso, 26, 46

Kato, M., \& Hachisu, I. 1994, ApJ, 437, 802

Leibowitz, E. M., \& Formiggini, L. 1992, A\&A, 265, 605

Mikolajewska, J., Kenyon, S. J., Mikolajewski, M., Garcia, M. R., \& Polidan, R. S. 1995, AJ, 109, 1289

Mikolajewska, J., \& Kenyon, S. J. 1992, MNRAS, 256, 177

Montagni, F., Maesano, M., Viotti, R., et al. 1996, IBVS, 4336

Mürset, U., Nussbaumer, H., Schmid, H. M., \& Vogel, M. 1991, A\&A, 248, 458

Nussbaumer, H., \& Vogel, M. 1987, A\&A, 182, 51

Nussbaumer, H., \& Vogel, M. 1988, in The Symbiotic Phenomenon, ed. J. Mikolajewska, M. Friedjung, S. J. Kenyon, \& R. Viotti (Kluwer), IAU Coll., 103169

Nussbaumer, H., \& Walder, R. 1993, A\&A, 278, 209

Petrik, K., Hric, L., Galis, R., Friedjung, M., \& Dobrotka A. 1998, IBVS, 4588

Schmid, H. M., Krautter, J., Appenzeller, I., et al. 1999, A\&A, 348,950

Seaton, M. J. 1979, MNRAS, 187, 73P

Shara, M. M., Prialnik, D., \& Kovetz, A. 1993, ApJ, 406, 220

Skopal, A. 1994, IBVS, 4096

Skopal, A. 1998, Contr. Astron. Obs. Skalnaté Pleso, 28, 87

Skopal, A., Hric, L., Chochol, D., et al. 1995, Contrib. Astron. Obs. Skalnaté Pleso, 25, 53

Smith, V. V., Cunha, K., Jorissen, A., \& Boffin, H. M. J. 1996, A\&A, 315, 179

Storey, P. J., \& Hummer, D. G. 1995, MNRAS, 272, 41

Tomov, N., \& Tomova, M. 2000, IBVS, 4953

Tomov, N., Tomova, M., \& Ivanova, A. 2000, A\&A, 364, 557

Tomova, M., \& Tomov, N. 1998, IBVS, 4561

Tomova, M., \& Tomov, N. 1999, A\&A, 347, 151

Vogel, M., \& Nussbaumer, H. 1994, A\&A, 284, 145

Yungelson, L., Livio, M., Tutukov, A., et al. 1995, ApJ, 447, 656 